their recent study, Lepore and colleagues have asked whether the drug shows similar benefits in patients with pulmonary hypertension secondary to congestive heart failure.

The study included 11 patients with leftventricular systolic dysfunction and pulmonary hypertension, evaluated for cardiac transplant at the Massachusetts General Hospital Heart Failure Service. During hemodynamic assessment, patients inhaled 80 ppm nitric oxide for $5 \mathrm{~min}$, received $50 \mathrm{mg}$ oral sildenafil $15 \mathrm{~min}$ later, and repeated the nitric oxide intake after $1 \mathrm{~h}$. Control patients underwent a similar procedure without sildenafil administration.

The combination of sildenafil and inhaled nitric oxide was associated with significant decreases in mean pulmonary artery pressure and systemic vascular resistance, and an increase in cardiac index. These changes were less pronounced when the two therapies were administered separately. Sildenafil extended the pulmonary vasodilatory effect of inhaled nitric oxide, apparently without affecting systemic arterial pressure or myocardial systolic or diastolic function.

In conclusion, these early results indicate that oral sildenafil improves cardiac output and increases the effects of nitric oxide inhalation in patients with severe congestive heart failure and pulmonary hypertension. If proven safe and effective in larger trials with clinical followup, sildenafil could be a valuable agent for the treatment of severe pulmonary hypertension in congestive heart failure patients.

Original article Lepore JJ et al. (2005) Hemodynamic effects of sildenafil in patients with congestive heart failure and pulmonary hypertension: combined administration with inhaled nitric oxide. Chest 127: 1647-1653

\section{Coronary circulatory dysfunction and insulin resistance}

A recent study by Prior et al. has investigated the link between insulin resistance and vascular injury. The results have shown that functional abnormalities in the coronary circulation appear early in the cascade of insulin resistance, and are more severe in individuals with carbohydrate intolerance.

The researchers used ${ }^{13} \mathrm{~N}$-ammonia and positron emission tomography to measure myocardial blood flow in 120 Mexican-American individuals, who had been divided into groups according to the presence or absence of insulin resistance, carbohydrate intolerance, diabetes and coronary risk factors. The procedure was performed at rest, during cold pressor testing, and during pharmacologic vasodilation.

Total vasodilator capacity was normal in normoglycemic individuals, including those with insulin resistance, carbohydrate intolerance or both. The onset of type 2 diabetes mellitus, however, was linked to a significant decrease in total vasodilator capacity, which was most pronounced in people with both diabetes and hypertension. By comparison with the insulin-sensitive individuals, those with insulin resistance showed significant deterioration in nitric-oxide-mediated, endothelium-dependent coronary vasomotion. The degree of impairment was greater in those with carbohydrate intolerance, and diabetes and hypertension were associated with even further deterioration.

The authors conclude that insulin resistance and carbohydrate intolerance carry a risk of progressive vascular injury and that this finding highlights the importance of diabetes prevention.

Original article Prior JO et al. (2005) Coronary circulatory dysfunction in insulin resistance, impaired glucose tolerance, and type 2 diabetes mellitus. Circulation 111:2291-2298

\section{Cardiovascular safety of NSAIDs: case-control analysis}

Nonsteroidal anti-inflammatory drugs (NSAIDs) are widely used for the relief of joint pain caused by arthritis. Within this class of drugs, cyclooxygenase 2 inhibitors have been developed to avoid the problem of gastrointestinal side effects. Recently, however, cyclo-oxygenase 2 inhibitors have been linked to an increased risk of cardiovascular complications-one such drug, rofecoxib, has been withdrawn from the market as a consequence. Two researchers from Nottingham University in the UK have now carried out a case-control study to examine the cardiovascular safety of rofecoxib and other NSAIDs.

Hippisley-Cox and Coupland analyzed use of NSAIDs among 9,218 patients with a first diagnosis of myocardial infarction between 2000 and 2004, and compared these results 